\title{
Anisotropic single-particle dissipative particle dynamics model
}

\author{
Mingge Deng ${ }^{\mathrm{a}}$ Wenxiao Pan ${ }^{\mathrm{b}}$ George Em Karniadakis ${ }^{\mathrm{a}}$

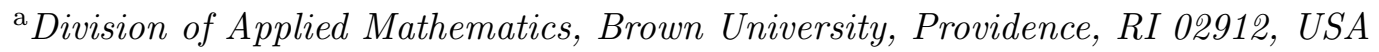 \\ ${ }^{\mathrm{b}}$ Department of Mechanical Engineering, University of Wisconsin-Madison, \\ Madison, WI 53706, USA
}

\begin{abstract}
We have developed a new single-particle dissipative particle dynamics (DPD) model for anisotropic particles with different shapes, e.g., prolate or oblate spheroids. In particular, the conservative and dissipative interactions between anisotropic single DPD particles are formulated using a linear mapping from the isotropic model of spherical particles. The proper mapping operator is constructed between each interacting pair of particles at every time step. Correspondingly, the random forces are properly formulated to satisfy the fluctuation-dissipation theorem (FDT). Notably, the model exactly conserves both linear and angular momentum. We demonstrate the proposed model's accuracy and efficiency by applying it for modeling colloidal ellipsoids. Specifically, we show it efficiently captures the static properties of suspensions of colloidal ellipsoids. The isotropic-nematic transition in an ellipsoidal suspension is reproduced by increasing its volume fraction or the aspect ratio of ellipsoid particles. Moreover, the hydrodynamics and diffusion of a single colloidal ellipsoid (prolate or oblate with moderate aspect ratios) are accurately captured. The calculated drag force on the ellipsoid and its diffusion coefficients (both translational and rotational) agree quantitatively with the theoretical predictions in the Stokes limit.
\end{abstract}

Key words:

Dissipative Particle Dynamics; Single-particle Dissipative Particle Dynamics; Colloid Suspension; Ellipsoidal Particle

Email address: wpan9@wisc.edu (Wenxiao Pan).

Preprint submitted to Journal of Computer Physics

13 January 2017

(C) 2017. This manuscript version is made available under the Elsevier user license http://www.elsevier.com/open-access/userlicense/1.0/ 


\section{Introduction}

The study of suspensions of Brownian colloids or nanoparticles encountered in biological and engineering applications has significantly advanced in recent years $[1-5]$. Colloidal particles can have a variety of shapes, including sphere, ellipsoid, rod, platelet, etc. A rather rich behavior of real colloidal systems can stem from the anisotropic shapes and interactions of colloidal particles [6-9]. Hence, the dynamics of anisotropic particles is fundamental in many associated physical phenomena, such as sedimentation, coagulation, migration, and non-Newtonian rheology. Therefore, accurate and efficient models for suspensions of anisotropic particles are needed in many practical applications. Clusters of multiple particles commonly have been used to represent a spherical colloid, stiff fiber, circular disc, or other bluff bodies in dissipative particle dynamics (DPD) and smoothed DPD simulations to capture the correct hydrodynamics [10-15]. However, such a representation may render a simulation prohibitively expensive.

Espanol et al. [16] proposed the fluid particle model (FPM) that shed lights in possibly modeling colloid particles as single DPD particles by including torques and angular velocities of particles into the model. Pan et al. [17] specified the general FPM formulation and demonstrated that the resulting model could capture the correct hydrodynamics of single DPD particles. The model was further extended to multi-sized particles to study colloidal suspensions [18], red blood cells [19], and also DNA [20]. However, all of those formulations were restricted to isotropic spherical particles. In the present work, we propose a new DPD formulation that allows for modeling anisotropic particles by single DPD particles. The new framework is applied to study both static and dynamic behaviors of colloidal ellipsoid suspensions, including microstructure, phase transition, and hydrodynamics, as well as translational and rotational diffusion.

This paper is organized as follows. Section 2 describes the governing equations, construction of the mapping operator, and formulations of the anisotropic force fields. Numerical results for different benchmark tests along with simulation strategy and parameters are presented and compared to analytical or theoretical predictions in Section 3. Finally, the paper concludes in Section 4 with a discussion about future applications of the proposed framework.

\section{Formulations}

We consider a canonical ensemble of single particles in three-dimensional (3D) space. The instantaneous state of the $i$ th particle with fixed mass $m_{i}$ and 
fixed-body frame inertia $\mathbf{I}_{i}^{c}$ is characterized by its position $\mathbf{r}_{i}(t)$, rotation matrix $\mathbf{R}_{i}(t)$, linear momentum $\mathbf{P}_{i}(t)$, and angular momentum $\mathbf{L}_{i}(t)$. These state variables provide the full spatial and velocity information. The $\mu$ th column ( $\mu=x, y, z)$ of $\mathbf{R}_{i}(t)$ represents the transformation of the $\mu$ th axis of a particle from its fixed-body frame to the laboratory frame, which makes the rotation matrix satisfy $\mathbf{R}^{T}=\mathbf{R}^{-1}$ and $|\mathbf{R}|=1$. We define a state vector as $\mathbf{Y}_{i}(t)=\left(\mathbf{r}_{i}(t), \mathbf{R}_{i}(t), \mathbf{P}_{i}(t), \mathbf{L}_{i}(t)\right)^{T}$, which is updated via the generalized Newton's second law at each time step as:

$$
\frac{d}{d t} \mathbf{Y}_{i}(t)=\left(\begin{array}{c}
\dot{\mathbf{r}}_{i}(t) \\
\dot{\mathbf{R}}_{i}(t) \\
\dot{\mathbf{P}}_{i}(t) \\
\dot{\mathbf{L}}_{i}(t)
\end{array}\right)=\left(\begin{array}{c}
\mathbf{v}_{i}(t) \\
\boldsymbol{\omega}_{i} \times \mathbf{R}_{i}(t) \\
\mathbf{F}_{i}(t) \\
\mathbf{N}_{i}(t)
\end{array}\right)
$$

Here, $\mathbf{v}_{i}(t)=m_{i}^{-1} \mathbf{P}_{i}(t), \boldsymbol{\omega}_{i}(t)=\mathbf{I}_{i}(t)^{-1} \mathbf{L}_{i}(t), \mathbf{F}_{i}(t)=\sum_{j \neq i} \mathbf{F}_{i j}$, and $\mathbf{N}_{i}(t)=$ $\sum_{j \neq i} \mathbf{N}_{i j}$, denoting the translational velocity, angular velocity, total force, and torque exerted on $i$ th particle at time $t$, respectively. $\mathbf{I}_{i}(t)$ is inertia in the laboratory frame and can be calculated via the rotation matrix as $\mathbf{I}_{i}(t)=$ $\mathbf{R}_{i}(t) \mathbf{I}_{i}^{c} \mathbf{R}_{i}(t)^{T}$

In the present work, only two-body pairwise interactions are considered in the calculation of the total force and torque. Furthermore, the pairwise forces satisfy Newton's third law, i.e., $\mathbf{F}_{i j}=-\mathbf{F}_{j i}$. As such, the total linear momentum $\mathbf{P}=\sum_{i} \mathbf{P}_{i}=\sum_{i} m_{i} \mathbf{v}_{i}$ is a dynamical invariant with $\dot{\mathbf{P}}=0$. In addition, the pairwise torque $\mathbf{N}_{i j}$ is defined as $\mathbf{N}_{i j}=-\mathbf{G}_{i j} \mathbf{r}_{i j} \times \mathbf{F}_{i j}$. Here, the prefactor matrix $\mathbf{G}_{i j}$ is included to account for the different contributions from interacting particles with different sizes and shapes. It can be considered a generalization of the scalar factor defined in the isotropic single-particle DPD method $[18,21]$ as a weight to account for the size difference of interacting particles. The specific form of $\mathbf{G}_{i j}$ will be determined later. When $\mathbf{G}_{i j}$ satisfies $\mathbf{G}_{i j}+\mathbf{G}_{j i}=\mathbf{I}$ (I: the $3 \times 3$ identity matrix), it immediately follows that the total angular momentum $\mathbf{J}=\sum_{i}\left(\mathbf{r}_{i} \times \mathbf{P}_{i}+\mathbf{I}_{i} \boldsymbol{\omega}_{i}\right)$ is also conserved, i.e., $\dot{\mathbf{J}}=0$.

The final expression of the corresponding Langevin equations for the examined particle system, in the form of stochastic differential equations, can be written as:

$$
\begin{aligned}
d \mathbf{r}_{i} & =\mathbf{v}_{i} d t=m_{i}^{-1} \mathbf{P}_{i} d t \\
d \mathbf{R}_{i} & =\boldsymbol{\omega}_{i} \times \mathbf{R}_{i} d t=\left(\mathbf{I}_{i}^{-1} \mathbf{L}_{i}\right) \times \mathbf{R}_{i} d t \\
d \mathbf{P}_{i} & =\sum_{j \neq i}\left[\left(\mathbf{F}_{i j}^{c}+\mathbf{F}_{i j}^{T}+\mathbf{F}_{i j}^{R}\right) d t+d \tilde{\mathbf{P}}_{i j}\right] \\
d \mathbf{L}_{i} & =-\sum_{j \neq i} \mathbf{G}_{i j} \mathbf{r}_{i j} \times\left[\left(\mathbf{F}_{i j}^{C}+\mathbf{F}_{i j}^{T}+\mathbf{F}_{i j}^{R}\right) d t+d \tilde{\mathbf{P}}_{i j}\right] .
\end{aligned}
$$

Here, as in Refs. [16,17] the deterministic forces between particle $i$ and $j$ consist 
of four different terms: the conservative force $\mathbf{F}_{i j}^{c}$, translational dissipative force $\mathbf{F}_{i j}^{T}$, rotational dissipative force $\mathbf{F}_{i j}^{R}$, and random force $\tilde{\mathbf{F}}_{i j}=d \tilde{\mathbf{P}}_{i j} / d t$. The last term compensates for the effect of eliminating degrees of freedom during the coarse-graining procedure and is formulated to satisfy the fluctuationdissipation theorem (FDT) given the expressions of the two dissipative forces. Previous studies $[17,18]$ have provided the specific formulations for the four force terms in the single-particle DPD model of isotropic spherical particles. In the present work, we aim to formulate the four force terms for slightly low symmetric (anisotropic) single particles in DPD.

To proceed, it is reasonable to assume that the mass density of an isotropic particle is Gaussian for computing the exclude-volume interactions, monopole, and dipole hydrodynamic forces between isotropic particles. Hence, for anisotropic particles, a generalized Gaussian function [22-24] is required. Specifically, if $\mathbf{M}$ can be expressed as a quadratic form matrix, the density distribution of a single particle centered at $\mathbf{r}_{0}$ can then be represented by a normalized Gaussian function as:

$$
\rho(\mathbf{r})=\frac{1}{\sqrt{8 \pi^{3}|\mathbf{M}|}} \exp \left[-\frac{1}{2}\left(\mathbf{r}-\mathbf{r}_{o}\right)^{T} \mathbf{M}^{-1}\left(\mathbf{r}-\mathbf{r}_{o}\right)\right]
$$

with $\mathbf{M}=\mathbf{R}^{T} \mathbf{S}^{2} \mathbf{R}$. Here, $\mathbf{S}$ is the shape matrix indicating the particle size and shape. For example, for an ellipsoid particle with semi-principal axes of length $R_{a}, R_{b}$, and $R_{c}, \mathrm{~S}=\operatorname{diag}\left(R_{a}, R_{b}, R_{c}\right)$. We particularly are interested in the interactions between two anisotropic particles $i$ and $j$, centered at $\mathbf{r}_{i}$ and $\mathbf{r}_{j}$, with the shape matrices $\mathbf{S}_{i}$ and $\mathbf{S}_{j}$, and the rotation matrices $\mathbf{R}_{i}, \mathbf{R}_{j}$. We denote

$$
\mathbf{r}_{i j}=\mathbf{r}_{i}-\mathbf{r}_{j}, \quad \mathbf{v}_{i j}=\mathbf{v}_{i}-\mathbf{v}_{j}, \quad \text { and } \quad \boldsymbol{\Omega}_{i j}=\boldsymbol{\omega}_{i} \times \mathbf{G}_{i j} \mathbf{r}_{i j}+\boldsymbol{\omega}_{j} \times \mathbf{G}_{j i} \mathbf{r}_{i j}
$$

Moreover, we define

$$
\mathbf{M}_{i}=\mathbf{R}_{i}^{T} \mathbf{S}_{i}^{2} \mathbf{R}_{i}, \quad \mathbf{M}_{j}=\mathbf{R}_{j}^{T} \mathbf{S}_{j}^{2} \mathbf{R}_{j}, \quad \text { and } \quad \mathbf{H}_{i j}=\mathbf{M}_{i}+\mathbf{M}_{j} .
$$

The prefactor matrix in the pairwise torque (Eq. 2) between particles $i$ and $j$ is thus determined as $\mathbf{G}_{i j}=\mathbf{M}_{i}^{-1}\left(\mathbf{M}_{i}^{-1}+\mathbf{M}_{j}^{-1}\right)^{-1}$, which exactly satisfies $\mathbf{G}_{i j}+\mathbf{G}_{j i}=\mathbf{I}$. Moreover, $\mathbf{H}_{i j}^{-1}$ is a real symmetric $3 \times 3$ matrix and can be decomposed as

$$
\mathbf{H}_{i j}^{-1}=\mathrm{OQ}^{2} \mathbf{O}^{T}=(\mathrm{OQ})(\mathrm{OQ})^{T} .
$$

The above decomposition is an eigendecomposition, in which the orthogonal matrix $\mathbf{O}$ is composed by the eigenvectors of $\mathbf{H}_{i j}^{-1}$, while $\mathbf{Q}^{2}$ is the diagonal matrix of eigenvalues. Here, $\mathbf{H}_{i j}^{-1}$ is a positive-definite matrix with all positive eigenvalues. The decomposition indicates both the rotational $(\mathbf{O})$ and elongational (Q) transformations from spherical to ellipsoidal shapes. Based on this decomposition, the linear mapping operator is constructed as (OQ), which 
thereby maps the anisotropic coordinates to isotropic ones as:

$$
\mathbf{r}_{i j}=(\mathrm{OQ}) \overline{\mathbf{r}}_{i j}, \quad \mathbf{v}_{i j}=(\mathrm{OQ}) \overline{\mathbf{v}}_{i j}, \quad \text { and } \quad \boldsymbol{\Omega}_{i j}=(\mathbf{O Q}) \bar{\Omega}_{i j}
$$

with $\bar{r}$ indicating the isotropic coordinates. Hence, a compact form for the deterministic forces between anisotropic DPD particles can be written as:

$$
\begin{aligned}
& \mathbf{F}_{i j}^{C}=(\mathbf{O Q}) a_{i j} W_{c}\left(\overline{\mathbf{r}}_{i j}\right) \overline{\mathbf{r}}_{i j} \\
& \mathbf{F}_{i j}^{T}=-(\mathbf{O Q}) W_{d}\left(\overline{\mathbf{r}}_{i j}\right)\left(\gamma_{i j}^{s} \mathbf{I}+\left(\gamma_{i j}^{c}-\gamma_{i j}^{s}\right) \overline{\mathbf{e}}_{i j} \otimes \overline{\mathbf{e}}_{i j}\right) \cdot \overline{\mathbf{v}}_{i j} \\
& \mathbf{F}_{i j}^{R}=-(\mathbf{O Q}) W_{d}\left(\overline{\mathbf{r}}_{i j}\right)\left(\gamma_{i j}^{s} \mathbf{I}+\left(\gamma_{i j}^{c}-\gamma_{i j}^{s}\right) \overline{\mathbf{e}}_{i j} \otimes \overline{\mathbf{e}}_{i j}\right) \cdot \overline{\mathbf{\Omega}}_{i j}
\end{aligned}
$$

with $\overline{\mathbf{e}}_{i j}=\overline{\mathbf{r}}_{i j} /\left|\overline{\mathbf{r}}_{i j}\right|$. Here, $a_{i j}, \gamma_{i j}^{c}$, and $\gamma_{i j}^{s}$ are the conservative, translational, and rotational dissipative coefficients, respectively, while $W_{c}$ and $W_{d}$ are the kernel functions for the respective conservative and dissipative forces. Instead of the widely used quadratic function, here, we adopt the Gaussian function with a parameter $\alpha$. The value of $\alpha$ indicates the rate of decay for different type of force. In particular, the Gaussian function for both $W_{c}$ and $W_{d}$ is given as:

$$
W\left(\overline{\mathbf{r}}_{i j}, \mathbf{H}_{i j}\right)=\frac{1}{\sqrt{\left|\mathbf{H}_{i j}\right|}} \exp \left(-\alpha \overline{\mathbf{r}}_{i j}^{T} \overline{\mathbf{r}}_{i j}\right),
$$

and can be obtained from the double integral of the Gaussian density distribution function in Eq. (3), i.e.,

$$
W\left(\mathbf{r}_{i j}, \mathbf{H}_{i j}\right)=\int d \mathbf{r}^{\prime} \int d \mathbf{r}^{\prime \prime} \rho_{i}\left(\mathbf{r}^{\prime}\right) \rho_{j}\left(\mathbf{r}^{\prime \prime}\right) \delta\left(\mathbf{r}^{\prime}-\mathbf{r}^{\prime \prime}\right)
$$

However, Eq. (5) can be replaced with other functions [22,24-29] by using different functions rather than the delta function in the above integral. Note that the parameter $\alpha$ in $W_{c}$ and $W_{d}$ generally takes different values. In particular, $\alpha_{c}$ in the conservative kernel $W_{c}$ is larger, reflecting the rapid decay of excluded-volume repulsive force, while $\alpha_{d}$ in the dissipative kernel $W_{d}$ is smaller, capturing the long-range nature of hydrodynamic dissipation. In the present work, we specifically use $\alpha_{c}=1.3$ and $\alpha_{d}=0.65$ throughout all of the simulations.

To illustrate the orientational anisotropy of the interacting forces, Fig. 1 shows the variation of the conservative forces between two identical ellipsoid particles with different distances and orientation angles. As the conservative force is essentially a repulsive excluded-volume interaction, the two ellipsoid particles tend to be parallel with each other to minimize their free energy at any given center-to-center distance.

Finally, the random force is formulated as:

$$
\tilde{\mathbf{F}}_{i j} d t=(\mathbf{O Q}) W_{r}\left(\overline{\mathbf{r}}_{i j}\right)\left[\frac{\sigma_{i j}^{c}}{\sqrt{3}} \operatorname{tr}\left[d \mathbf{W}_{i j}\right] \mathbf{I}+\sqrt{2} \sigma_{i j}^{s} d \mathbf{W}_{i j}^{A}\right] \cdot \overline{\mathbf{e}}_{i j} .
$$




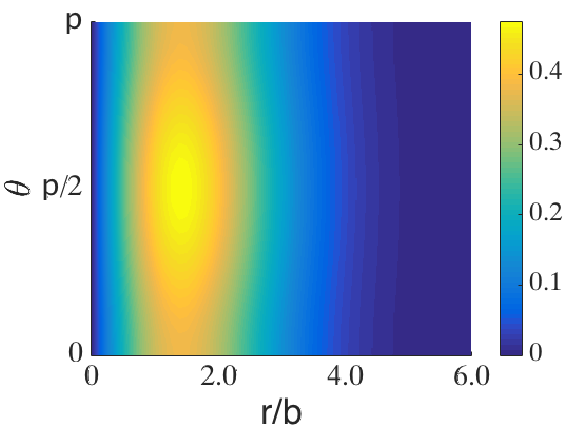

(a)

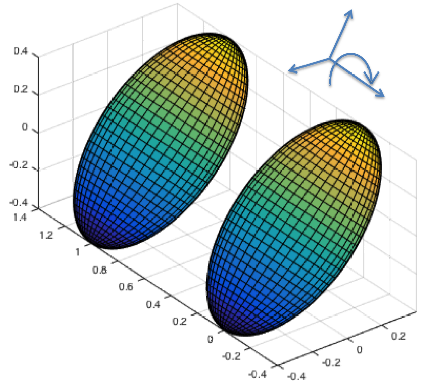

(b)

Fig. 1. (a) The magnitude contour of conservative forces between two identical ellipsoid particles with semi-axis lengths of $0.8,0.4$, and 0.4 , at varying distances and orientation angles. Here, one particle is fixed, while the other particle rotates around one of its short axes with a varying angle $\theta$. The two ellipsoid particles are initially positioned parallel along the short axes as sketched in (b).

Here, $d \mathbf{W}_{i j}$ is a random matrix of independent Wiener increments, which is symmetric under particle interchange. $d \mathbf{W}_{i j}^{A}$ is the antisymmetric random matrix with each element defined as $d \mathbf{W}_{i j}^{A \mu \nu}=\frac{1}{2}\left(d \mathbf{W}_{i j}^{\mu \nu}-d \mathbf{W}_{i j}^{\nu \mu}\right)$. The random force coefficients are $\sigma_{i j}^{s}=\sqrt{2 k_{B} T \gamma_{i j}^{s}}$ and $\sigma_{i j}^{c}=\sqrt{2 k_{B} T \gamma_{i j}^{c}}$. The kernel function in the random force satisfies $W_{r}^{2}\left(\overline{\mathbf{r}}_{i j}\right)=W_{d}\left(\overline{\mathbf{r}}_{i j}\right)$. Based on this and due to the invariant property of linear mapping, the FDT automatically is satisfied, which also is verified numerically by examining the kinetic temperature in each translational and rotational degree of freedom (refer to next section).

In summary, we have formulated the anisotropic single-particle DPD model with tunable effective parameters $a_{i j}, \gamma_{i j}^{s}, \gamma_{i j}^{c}, \alpha_{c}$, and $\alpha_{d}$. The new formulation essentially is constructed via linear transformation from isotropic spherical interactions to anisotropic ones, and it can be readily reduced to that in the isotropic single-particle DPD model [17]. In the next section, the proposed framework is applied to study the static and dynamic properties of single ellipsoid particle (dilute suspension) and concentrated ellipsoid suspensions.

\section{Numerical Results}

In all simulations presented in this section, the ellipsoid suspension model consists of suspended colloids represented as single ellipsoid DPD particles with a larger dimension and mass, as well as a solvent bath of isotropic spherical DPD particles with a smaller radius and mass. Specifically, the solvent particle has a constant radius of 0.25 , while the semi-axis lengths $\left(R_{a}, R_{b}\right.$, and $\left.R_{c}\right)$ of the colloidal particle vary around 1.0, all in reduced DPD units. Without loss of generality, we consider ellipsoids with a pair of equal semi-axes and 
a distinct third semi-axis, i.e., prolate spheroids (two equal short axes and a long axis) and oblate spheroids (two equal long axes and one short axis). The simulation domain is $20 \times 40 \times 20$, and the number density of solvent particles $\rho=4$. First, the initial positions and orientations of DPD particles are randomly assigned. Next, a preliminary no-flow simulation is conducted with a very small timestep for the system to reach equilibrium and avoid overlap between DPD particles. The velocity-verlet time-integration scheme is used to update both translational and rotational dynamics of DPD particles. The temperature is not sensitive to the time step when $d t<0.1$. Both the linear and angular momenta are conserved, and each follows the Boltzmann distribution, as discussed later.

\subsection{Solvation of a single ellipsoid}

First, we examine the solvation of a single colloid immersed in solvent at equilibrium. Fig. 2 depicts the impenetrable area of the colloidal particle by the

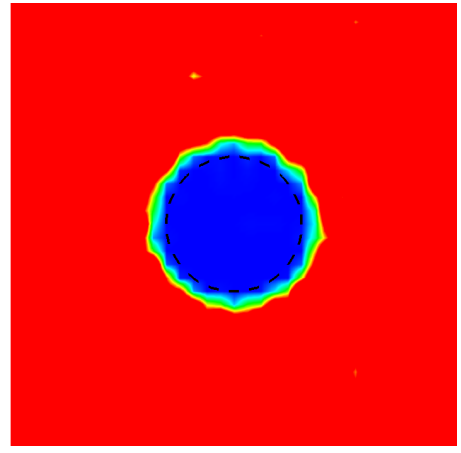

(a)

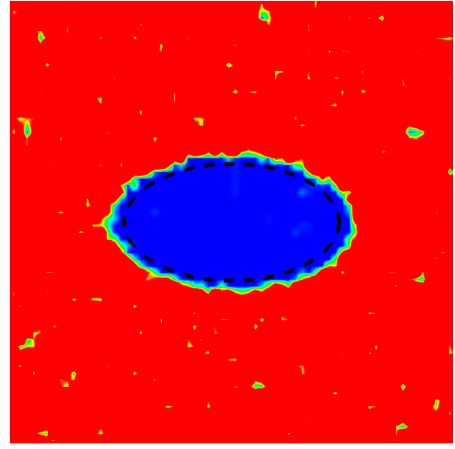

(b)

Fig. 2. Number density distribution of solvent particles with a solvation layer around an immersed single (a) spherical particle with a radius of 1.0, and (b) ellipsoid particle with semi-axis lengths of $1.5,0.8165$, and 0.8165 . The black dash lines indicate the colloid-intrinsic boundaries.

number density distribution of its surrounding solvent particles. By adjusting the parameters of conservative interactions between particles (solvent-solvent (ss), colloid-solvent (cs), and colloid-colloid (cc)), i.e., $a_{s s}=2.5, a_{c s}=175.2$, and $\alpha_{c}=1.30$, the calculated shape and dimensions for the impenetrable area of the colloidal particle are found to be consistent with the intrinsic shape and dimensions of the colloid as the input parameters in the shape matrix. This indicates that our conservative force can effectively preserve the size and shape of an anisotropic particle. The results depicted in Fig. 2 are obtained under the zero-flow condition. Similarly as the fluid modeled by DPD is weakly compressible, the colloidal particles described by our current model are also weakly deformable. Thus, slight deformations are expected for large Peclet number $(P e)$ and Reynolds number $(R e)$. However, within the 
parameter range throughout the simulations in this paper, i.e. $P e<<1$ and $R e<<1$, no obvious deformation in particles' shape is observed, as indicated by the mean density profile of surrounding solvent particles.

\subsection{Microstructure in ellipsoid suspensions}

Second, we use the proposed new DPD model to study the microstructure of colloidal ellipsoids in suspensions. Recently, Cohen and coworkers reported that compared to isotropic spherical colloids, the rotational anisotropy of ellipsoids tends to smear out the shell structures of colloids in suspensions with moderate volume fractions [30]. This can be examined by computing the radial distribution function (RDF) $g(r)$ of colloidal particles. As shown in Fig. 3,

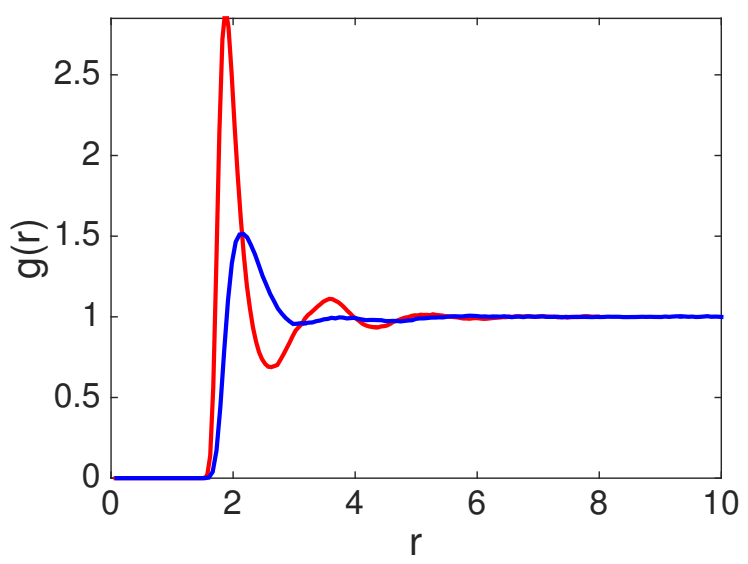

Fig. 3. Radial distribution function $g(r)$ of prolate ellipsoids $\left(R_{b}=R_{c}\right)$ with the aspect ratio $R_{a} / R_{b}=2.0$ (blue line) and spherical colloids (red line) in suspensions with the same volume fraction $\phi=0.31$. Here, $r$ is normalized by the short axis length $\left(R_{b}\right)$ of prolate for ellipsoids or by the radius of sphere $\left(R_{s}\right)$ for spherical colloids.

the peaks in the computed $g(r)$ of ellipsoids are much less pronounced than those of spherical colloids. This finding is consistent with the direct structural measurements by Cohen et al. [30]. Thus, our model accurately captures the microstructure of colloidal ellipsoids in suspensions. We note that $g(r)$ is not identically zero for $r<2$, reflecting the fact that colloidal particles slightly overlap due to the soft Gaussian kernel function (Eq. 5). In addition, the excluded volume are the same for the sphere and ellipsoid. However, spheres prevent the slight overlap better than ellipsoids, which is indicated by the positions of the first peaks in the plot of $g(r)$ (Fig. 3). This is because the force is stiffer when two ellipsoids are perpendicular to each other but softer along the parallel directions, allowing for more overlap along parallel directions for ellipsoids. 
The isotropic-nematic transition is another interesting phenomenon for concentrate suspensions of colloidal ellipsoids. In the isotropic phase, colloidal particles are randomly distributed without any orientational order. As the volume fraction of ellipsoids or the anisotropy of ellipsoids increases, the transition from the isotropic to nematic phase takes place, in which colloidal particles tend to align in parallel to minimize the free energy of repulsive interactions.

Fig. 4 (a) and (b) show the snapshots of both phases for ellipsoid particles

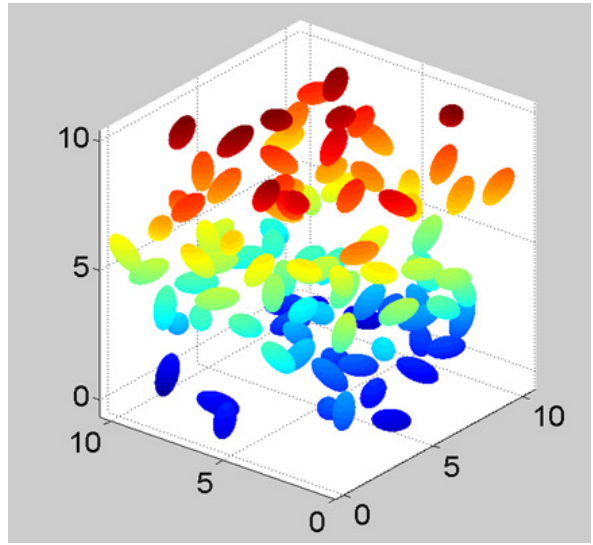

(a)

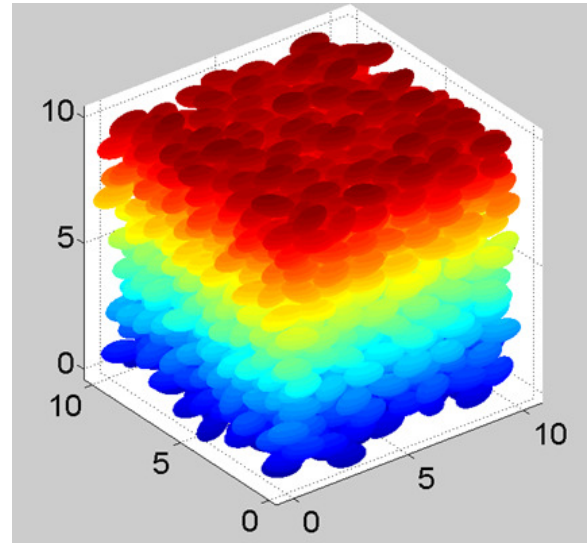

(b)

Fig. 4. Microstructures of colloidal ellipsoids in suspensions corresponding to the isotropic-nematic phase transition with an increasing volume fraction: (a) isotropic phase, $\phi=5.3 \%, R_{a} / R_{b}=2.0$; (b) nematic phase, $\phi=70 \%, R_{a} / R_{b}=2.0$.

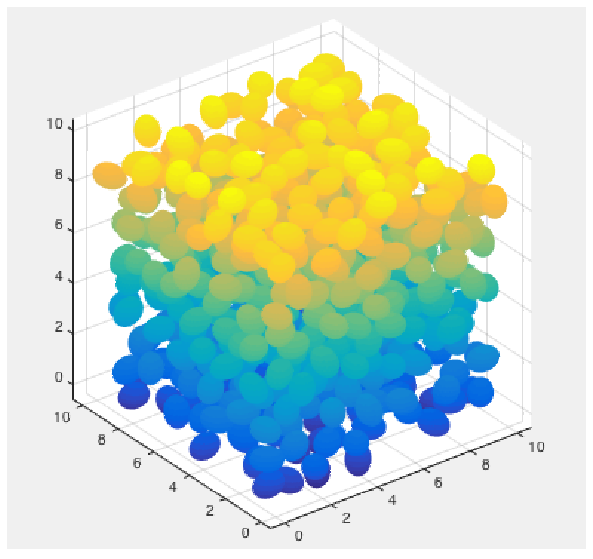

(a)

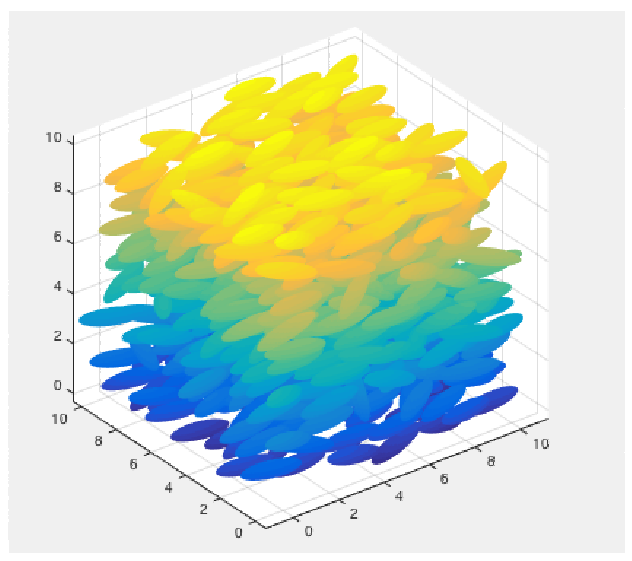

(b)

Fig. 5. Microstructures of colloidal ellipsoids in suspensions corresponding to the isotropic-nematic phase transition with an increasing aspect ratio: (a) isotropic phase, $\phi=31 \%, R_{a} / R_{b}=1.31$; (b) nematic phase, $\phi=31 \%, R_{a} / R_{b}=3.71$.

with the same aspect ratio at different volume fractions. While Fig. 5 (a) and (b) illustrate the phase transition from one to the other due to the increased aspect ratio of ellipsoids at the same volume fraction. This phase transition can be characterized by the orientation order parameter $S$, which, by defini- 
tion, is the largest eigenvalue of the second-rank ordering tensor $\boldsymbol{\Lambda}$ that can be calculated from

$$
\boldsymbol{\Lambda}_{\nu \mu}=<\frac{1}{N} \sum_{i}^{N} \frac{3}{2} \hat{\mathbf{n}}_{i \nu} \hat{\mathbf{n}}_{i \mu}>-\frac{1}{2} \delta_{\nu \mu}
$$

Here, $\hat{\mathbf{n}}$ is the unit vector along the ellipsoid long axis in the laboratory frame, and $\nu, \mu$ vary between $x, y$, and $z$. Fig. 6 (a) depicts the variation of $S$

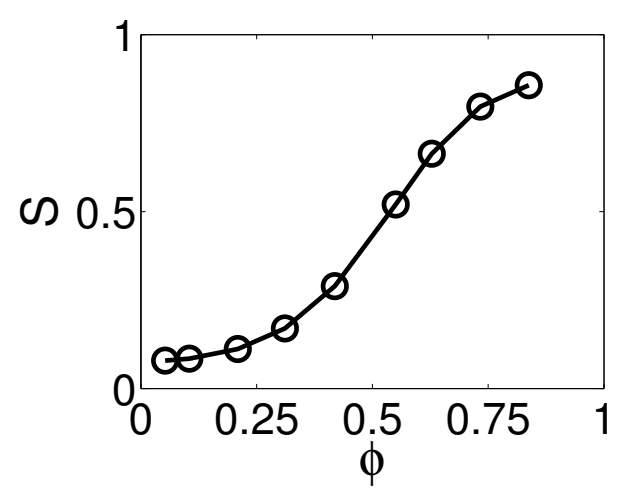

(a)

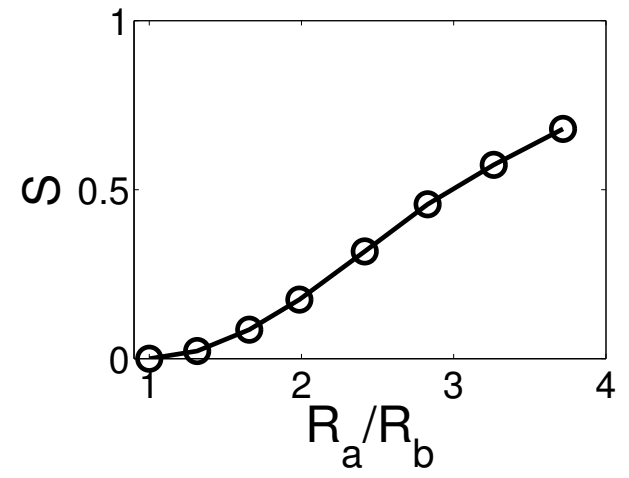

(b)

Fig. 6. The orientation order parameter $S$ (a) as a function of volume fraction $\phi$ at $R_{a} / R_{b}=2.0$ and (b) as a function of $R_{a} / R_{b}$ at $\phi=0.31$. The solid line acts as a guide.

as a function of the volume fraction $\phi$, given the fixed ellipsoid aspect ratio $R_{a} / R_{b}=2.0$. In this example, $S$ is near zero when $\phi$ is small, and the transition takes place when $\phi$ reaches $50 \%-60 \%$, followed by a gradual increase toward unity as $\phi$ further increases. The transition point at about $50 \%-60 \%$ is found to agree with previous studies $[31,32]$. However, we note that the transition is not as sharp as those predicted in the Percus-Yevick theory [31] or the Gay-Berne potential-based molecular dynamics simulation [33,32], which can result from the soft Gaussian-type potential used in the model. In Fig. 6 (b), varying $S$ is associated with the isotropic-nematic transition due to the increased aspect ratios $R_{a} / R_{b}$, even at a moderate volume fraction, i.e., $\phi=$ $31 \%$.

\subsection{Thermostat}

In this section, the thermostat in the new anisotropic DPD model is verified by checking the translational, rotational, and total kinetic temperatures of a homogeneous particle system consisting of identical ellipsoidal DPD particles. In Fig. 7 (a), the new DPD thermostat robustly yields fast convergence for all kinetic temperatures to their desire values, indicating that the FDT is accurately satisfied. In addition, Fig. 7 (b) depicts that total energy, including 
kinetic and conservative energies, converges fast to its equilibrium value. Moreover, both the linear and angular momenta are found to follow the Boltzmann distribution precisely, which are illustrated in Fig. 7 (c) and (d).

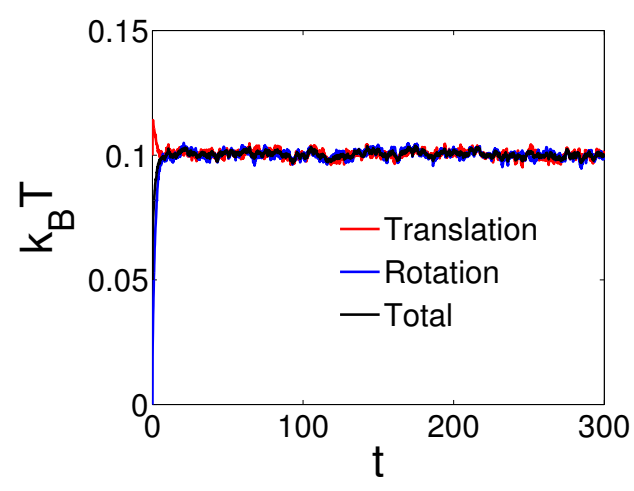

(a)

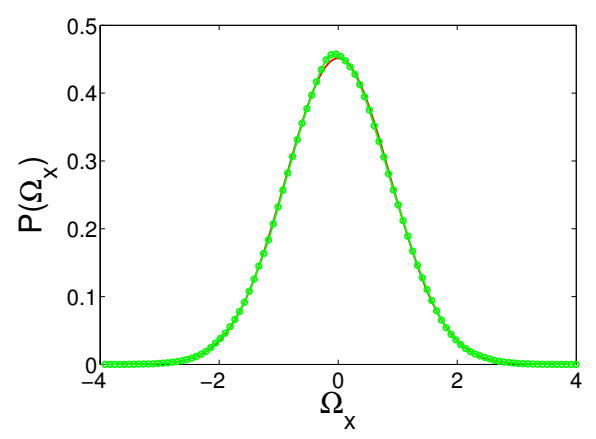

(c)

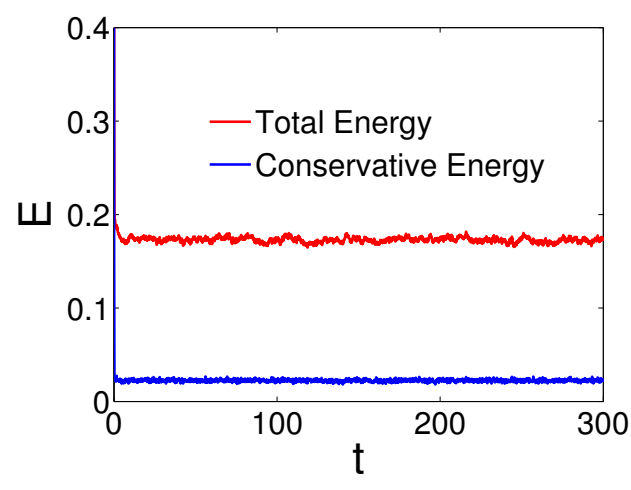

(b)

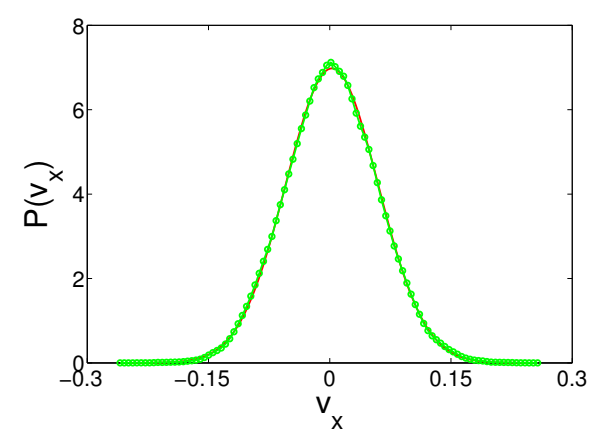

(d)

Fig. 7. (a) Kinetic temperatures and (b) energies converge to their equilibrium values in a homogeneous particle system consisting of identical ellipsoidal DPD particles; (c) angular and (d) translational velocities follow the Boltzmann distribution.

\subsection{Hydrodynamics of an ellipsoid in suspension}

Notably, DPD was invented to accurately capture the dynamic properties of coarse-grained systems, including hydrodynamics and diffusion, which is the primary advantage of DPD compared to other coarse-grained methods. Thus, we examine the proposed model's accuracy in reproducing the correct hydrodynamics and diffusion of a colloidal ellipsoid in suspension.

We first study the hydrodynamics of flow around ellipsoid DPD particles. In particular, we test a uniform flow past a periodic array of oblate ellipsoids. The oblate ellipsoid is held stationary at the center of a cubic periodic domain. The flow then is driven by an external body force $F_{\text {ext }}$ exerted on all solvent particles, and the flow direction is along one of the ellipsoid's short axes. The 
cubic lattice array was sized to ensure that the effect of the periodic neighboring colloids is negligible, which was verified by doubling the lattice dimension. Fig. 8 (a) and (b) show the calculated velocity fields around the colloid along the flow direction for different-shaped oblate ellipsoids for a small portion of the cubic domain. The drag force $F_{d}$ exerted on the stationary particle is com-

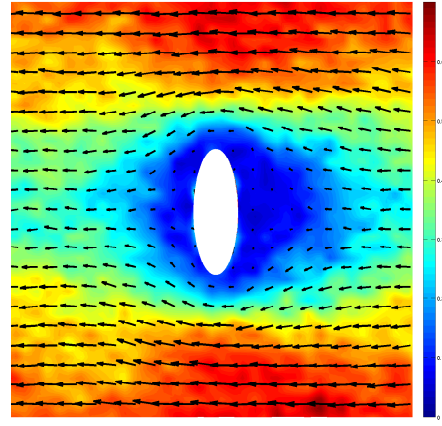

(a)

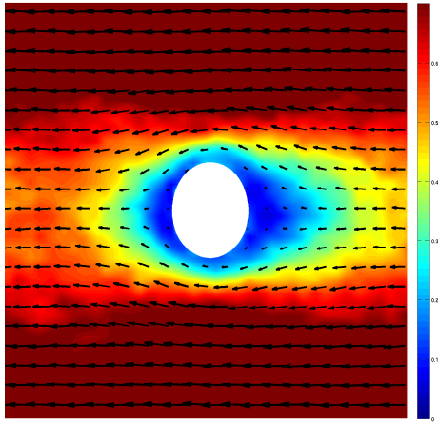

(b)

Fig. 8. The velocity field of uniform flow past a periodic array of oblate ellipsoids with semi-axis lengths of (a) 1.414, 1.414, and 0.5; (b) 1.085, 1.085, and 0.85.

puted, and for the solvent particles, an average superficial streaming velocity $U$ is calculated from $U=\frac{1}{V} \int_{V} u(x, y, z) d V$. At low Reynolds numbers, the drag force on the oblate satisfies the Stokes law: $F_{d}=6 \beta \pi \eta U R_{s}$. Here, $R_{s}$ is the effective Stokes radius of the oblate, and $\beta$ is the shape factor. The

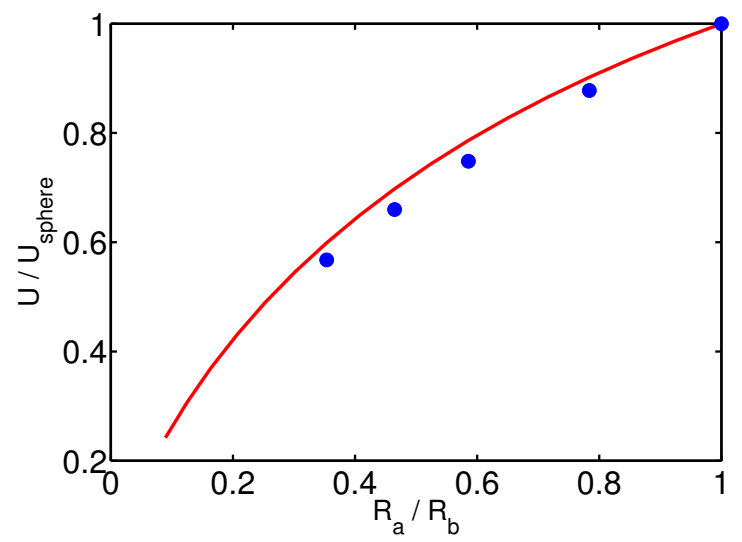

Fig. 9. The calculated relative superficial velocity of flow around an oblate ellipsoid as a function of aspect ratio $R_{a} / R_{b}$ (symbol), compared with the analytical results [34] (solid line).

calculated superficial velocity as a function of the oblate aspect ratio is plotted in Fig. 9 and compared with the analytical prediction from the Stokes law [34]. Here, the calculated superficial velocities are normalized by that of the flow around a spherical colloid with the same volume and body force $F_{\text {ext }}$ exerted on solvent particles. Throughout the simulations, we first consider a 
pure solvent composed of isotropic spherical DPD particles with parameters: $a_{s s}=2.5, \gamma_{s s}^{c}=\gamma_{s s}^{s}=0.25, \alpha_{c}=1.30, \alpha_{d}=0.65$, and $r_{c}=1.0$. Here, the cutoff radius $r_{c}$ is selected such that the kernel vanishes beyond the cutoff distance. The solvent viscosity is computed from the reverse Poiseuille flow as $\eta=1.64$. Next, we consider the suspension with a single spherical colloid. By adjusting the parameter $a_{c s}$ and cutoff radius in the conservative force between the solvent and colloidal particles, its effective geometrical radius $R_{c}$ (measured from the mean density profile of its surrounding solvent particles as shown in Fig. 2) matches the input radius of the sphere. Then, we adjust the parameters $\gamma_{c s}^{c}$ and $\gamma_{c s}^{s}$ in the dissipative force between the solvent and colloidal particles to reproduce the correct hydrodynamics, i.e., making its hydrodynamic radius $R_{s}$ (measured from the Stokes' law with the drag force) equal to $R_{c}$. Thus far, these parameters are determined as: $a_{c s}=160.0, \gamma_{c s}^{c}=8.0, \gamma_{c s}^{s}=9.0$, and $r_{c}=2.5$. After this procedure, we applied the linear mappings for simulating the flow past an ellipsoidal colloid without any further calibration of parameters. We expect the hydrodynamic radius of the ellipsoid is equal to that of the sphere with the same volume, thus, the drag force or the mean velocity of the suspended colloid only depends on the shape factor here.

\subsection{Diffusion of an ellipsoid in suspension}

Having demonstrated that the model can accurately capture the hydrodynamics of colloidal ellipsoid in flow, we next apply it to study the diffusion of ellipsoid particles. Diffusion of an ellipsoid particle can be described by its translational and angular mean-square displacements (MSDs), as previously studied by Han et al. [35,36] for quasi-two-dimensional (2D) ellipsoids.

In the simulations, we record the ellipsoid particle trajectory in the laboratory frame, including the center of mass position $\mathbf{r}(t)$ and rotation matrix $\mathbf{R}(t)$ at each time step. At the $n$th time step, the particle's translational displacement $\delta \mathbf{r}\left(t_{n}\right)$ is mapped onto the fixed-body frame, i.e., obtaining the translational displacement along the principal axes denoted as $\delta \tilde{\mathbf{r}}\left(t_{n}\right)$ by $\delta \tilde{\mathbf{r}}\left(t_{n}\right)=\mathbf{R}\left(t_{n}\right) \delta \mathbf{r}\left(t_{n}\right)$. The rotational displacement around the principal axes $\delta \tilde{\boldsymbol{\theta}}\left(t_{n}\right)$ is approximated by the infinitesimal rotation matrix as $\mathbf{e}=\mathbf{R}\left(t_{n+1}\right) \mathbf{R}^{-1}\left(t_{n}\right)-\mathbf{I}$, which is antisymmetric and has the form of

$$
\mathbf{e}=\left(\begin{array}{ccc}
0 & \delta \tilde{\theta}_{c} & -\delta \tilde{\theta}_{b} \\
-\delta \tilde{\theta}_{c} & 0 & \delta \tilde{\theta}_{a} \\
\delta \tilde{\theta}_{b} & -\delta \tilde{\theta}_{a} & 0
\end{array}\right)
$$

Thus, the total fixed-body frame displacements after $n$ time steps is the summation of all displacements in each time step, i.e., $\tilde{\mathbf{r}}\left(t_{n}\right)=\sum_{k=1}^{n} \delta \tilde{\mathbf{r}}_{k}$, and 
$\tilde{\boldsymbol{\theta}}\left(t_{n}\right)=\sum_{k=1}^{n} \delta \tilde{\boldsymbol{\theta}}_{k}$. Then, the displacements in the fixed-body frame of any duration $t$ at a starting time $\tau_{0}$ are calculated as $\Delta \tilde{\mathbf{r}}(t)=\tilde{\mathbf{r}}\left(t+\tau_{0}\right)-\tilde{\mathbf{r}}\left(\tau_{0}\right)$ and $\Delta \tilde{\boldsymbol{\theta}}(t)=\tilde{\boldsymbol{\theta}}\left(t+\tau_{0}\right)-\tilde{\boldsymbol{\theta}}\left(\tau_{0}\right)$, respectively. Fig. 10 (a) and (b) depict the

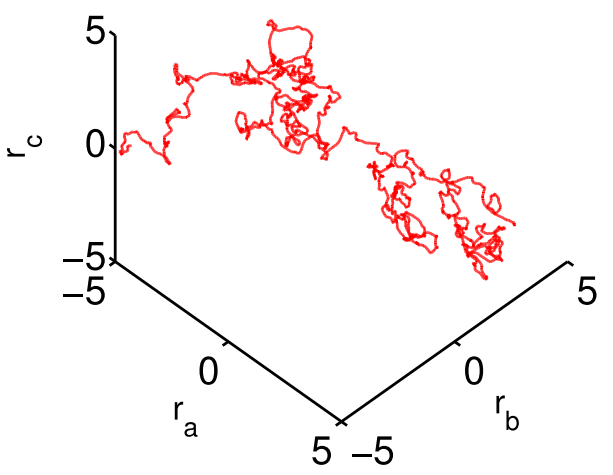

(a)

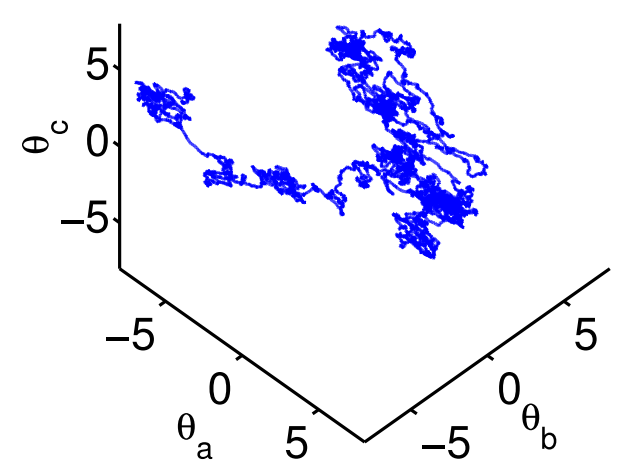

(b)

Fig. 10. (a) Translational and (b) rotational trajectories of an ellipsoid particle in the fixed-body frame with semi-axis lengths of $0.8,0.4$, and 0.4 .

calculated translational and rotational trajectories of an ellipsoid particle in the fixed-body frame. As anticipated, the diffusion of an anisotropic particle varies with respect to direction. Specifically, diffusion is significantly faster along the long axis than along the two short axes.

The MSDs in the fixed-body frame are statistically averaged (ensemble average) over all trajectories and different starting times $\tau_{0}$. They follow normal diffusion laws in long-time limit as

$$
<\left[\Delta \tilde{r}_{\mu}(t)\right]^{2}>=2 D_{t \mu} t, \quad \text { and } \quad<\left[\Delta \tilde{\theta}_{\mu}(t)\right]^{2}>=2 D_{r \mu} t,
$$

with $\mu=a, b, c$ (axes in the fixed-body frame). We further define the overall average translational self-diffusion coefficient as

$$
D_{t}=\left(D_{t a}+D_{t b}+D_{t c}\right) / 3
$$

and the average rotational self-diffusion coefficient as

$$
D_{r}=\left(D_{r a}+D_{r b}+D_{r c}\right) / 3 .
$$

The self-diffusion coefficients of a colloidal particle are related to its size and shape (effective hydrodynamical radius) via the Stokes-Einstein relation. Fig. 11 (a) and (b) illustrate the computed MSDs in the fixed-body frame of a single ellipsoid with the aspect ratio $R_{a} / R_{b}=2.0$. The ratio between the translational diffusion coefficients along the long and short axes, i.e., $D_{t a} / D_{t b}$ 


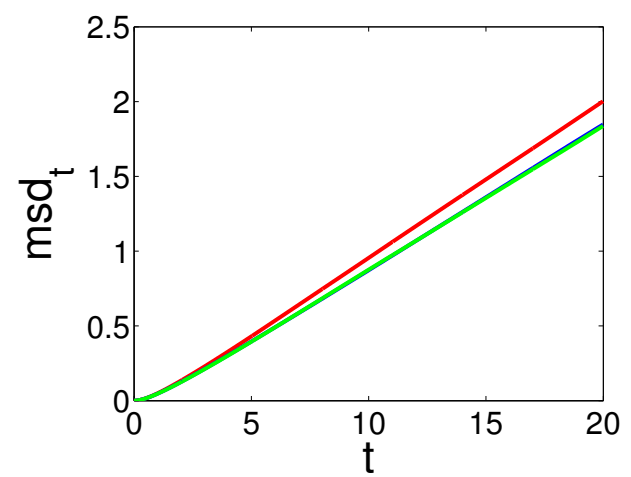

(a)

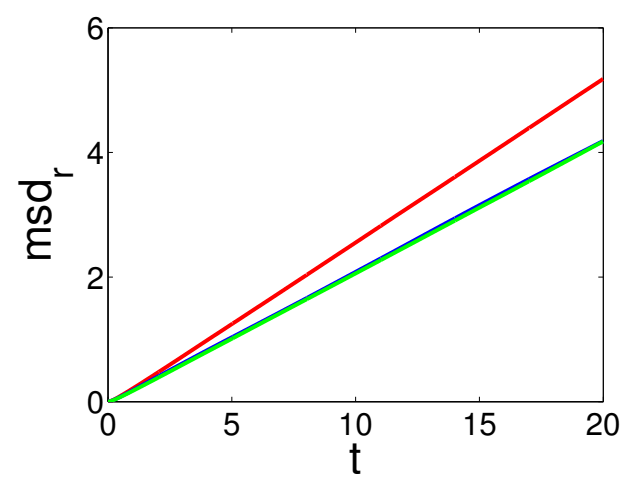

(b)

Fig. 11. (a) Translational MSDs of an ellipsoid particle with semi-axis lengths of $0.8,0.4$, and 0.4 along the fixed-body long axis (red) and short axis (green). (b) Rotational MSDs around the fixed-body long axis (red) and the short axis (green).

$\left(D_{t b}=D_{t c}\right)$, is estimated to be about 1.20 in our simulation, close to the theoretical result of about 1.15 [37]. The ratio between the rotational diffusion coefficients around the long and short axes, i.e., $D_{r a} / D_{r b}\left(D_{r b}=D_{r c}\right)$, is about 1.27 .

The translational $\left(D_{t}\right)$ and rotational diffusions $\left(D_{r}\right)$ of a prolate particle relative to the corresponding diffusions $\left(D_{t}^{0}, D_{r}^{0}\right)$ of a spherical particle with the same volume have been well established by Perrin [38,39]. Therefore, we

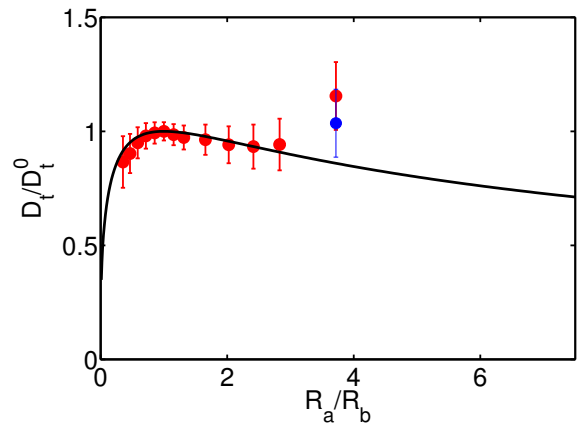

(a)

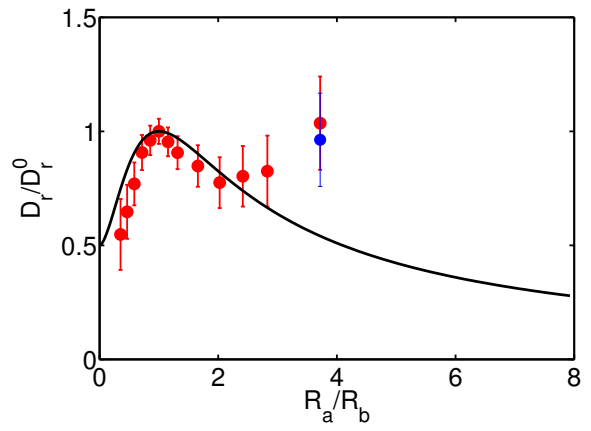

(b)

Fig. 12. (a) The relative translational and (b) rotational diffusion coefficients as a function of aspect ratios (symbol), compared with Perrin's analytical results (black solid line). Error bars are calculated as standard deviations of the ensemble average.

plot the normalized $D_{t}$ and $D_{r}$ by $D_{t}^{0}$ and $D_{r}^{0}$, respectively, as a function of the aspect ratio in Fig. 12 (a) and (b). Our results agree well with Perrin's analytical results for up to moderate aspect ratios, i.e., $R_{a} / R_{b} \in(0.3,3.5)$. As the anisotropy becomes more pronounced, leading to a very elongated ellipsoid, the simulation results begin to deviate from the analytical prediction. This can be alleviated by increasing the simulation resolution using smaller solvent particles, which results in more expensive computational cost. As shown in 
Fig. 12 (a) and (b), the agreement with the analytical results improves using a doubled number density of solvent particles (blue dots). In practice, we also can make the dissipative parameters of anisotropic particles adaptive to their aspect ratios, instead of fixing their values as those of a spherical particle with the same volume.

\section{Discussion}

An efficient new computational method has been developed for modeling anisotropic bluff bodies using single particles in DPD, filling a gap in the current literature on mesoscopic methods. We have demonstrated the proposed method's accuracy by capturing both static and dynamic properties of suspensions of colloidal ellipsoids. With regards to computational cost, in the present DPD model the computational cost is proportional to the total number of particles in the system $(O(N))$, which is about 10 times the number of suspended colloids, $N_{c}$, for an average volume fraction. Hence, the speed-up factor compared to the standard DPD method typically using 200 DPD particles for each suspended colloid is a factor of about 20. Likewise, in the isotropic single-particle DPD model, to represent an anisotropic colloid a certain number (e.g., several tens) of DPD particles are needed. Thus, the computational efficiency of the present model compared to both the standard DPD and previously developed isotropic single-particle DPD models is remarkable. Three remarks bring further clarity to this new approach. First, the soft Gaussian excluded-volume repulsion between single DPD particles can be replaced by some hard potentials, such as the empirical Gay-Berne [27] and RE-squared [26] potentials used in molecular dynamics simulations, and other specifically-designed interaction potentials, which will make this model attractive to simulate self-assembly of nanoparticles in complex materials [40]. Additionally, the lubrication force is not considered in the general DPD framework for various reasons, for example, the short-range lubrication force (hydrodynamic force) is stiff, which is beyond DPD (soft interactions) applicable range. Thus, without further description of lubrication force, the application of current model to the dynamics of colloidal suspensions at large volume fraction and Peclet number $(P e>1)$ is limited and out of the scope of this work. Second, to model particles with strong anisotropy, higher resolutions and hence more expensive computations are necessary to resolve the singularities at the sharp ends of particles. Alternatively, low-symmetric bluff bodies can be modeled as clusters of small anisotropic particles. Hence, we can extend our model to study both static and dynamic properties of suspensions of a broad range of low-symmetric bluff bodies, such as boomerang colloidal particles [41] that can be effectively constructed via two ellipsoids. Lastly, the present framework generalizes the standard isotropic DPD model to afford development of more 
efficient anisotropic coarse-grained models for macromolecules and proteins [42].

\section{Acknowledgments}

This work was supported by the Applied Mathematics Program within the U.S. Department of Energy (DOE) Office of Advanced Scientific Computing Research (ASCR) as part of the Collaboratory on Mathematics for Mesoscopic Modeling of Materials (CM4). Computations were performed using a DOE INCITE award.

\section{References}

[1] V. Wagner, A. Dullaart, A. Bock, A. Zweck, The emerging nanomedicine landscape, Nat. Biotechnol. 24 (2006) 1211-1217.

[2] P. Burgess, P. B. Hutt, O. C. Farokhzad, R. Langer, S. Minick, S. Zale, On firm ground: IP protection of therapeutic nanoparticles, Nat. Biotechnol. 28 (2010) $1267-1270$.

[3] L. Y. Rizzo, B. Theek, G. Storm, F. Kiessling, T. Lammers, Recent progress in nanomedicine: therapeutic, diagnostic and theranostic applications, Curr. Opin. Biotechnol. 24 (2013) 1159-1166.

[4] K. Tiede, A. Boxall, S. Tear, J. Lewis, H. David, M. Hassellov, Detection and characterization of engineered nanoparticles in food and the environment, Food Addit Contam 25 (2008) 795-821.

[5] M. Hochella, Nanoscience and technology: the next revolution in the earth sciences, Earth Planet Sci. Lett. 203 (2002) 593-605.

[6] S. Sacanna, D. Pine, Shape-anisotropic colloids: Building blocks for complex assemblies, Curr. Opin. Colloid Interface Sci. 16 (2011) 96-105.

[7] S. Glotzer, M. Solomon, Anisotropy of building blocks and their assembly into complex structures, Nat. Mater. 6 (2007) 557-562.

[8] S. Yang, S. Kim, J. Lim, G. Yi., Synthesis and assembly of structured colloidal particles, J. Mater. Chem. 18 (2008) 2177-2190.

[9] Z. Mao, H. Xu, D. Wang, Molecular mimetic self-assembly of colloidal particles, Adv. Funct. Mater. 20 (2010) 1053-1074.

[10] E. S. Boek, P. V. Coveney, H. N. W. Lekkerkerker, P. van der Schoot, Simulating the rheology of dense colloidal suspensions using dissipative particle dynamics, Phy. Rev. E 55 (3) (1997) 3124-3133. 
[11] N. S. Martys, Study of a dissipative particle dynamics based approach for modeling suspensions, J. Rheol. 49 (2) (2005) 401-424.

[12] N. Phan-Thien, N. Mai-Duy, B. C. Khoo, A spring model for suspended particles in dissipative particle dynamics, J. Rheol. 58 (2014) 839.

[13] N. Mai-Duy, N. Phan-Thien, B. Khoo, Investigation of particles size effects in dissipative particle dynamics (DPD) modelling of colloidal suspensions, Comput. Phys. Commun. 189 (2015) 37.

[14] X. Bian, S. Litvinov, R. Qian, M. Ellero, N. Adams, Multiscale modeling of particle in suspension with smoothed dissipative particle dynamics, Phys. Fluids 24 (2012) 012002.

[15] A. Vazquez-Quesada, M. Ellero, P. Espanol, A SPH-based particle model for computational microrheology, Microfluid Nanofluidics 13 (2012) 249.

[16] P. Espanol, Fluid particle model, Phys. Rev. E 57 (1998) 2930-2948.

[17] W. Pan, I. Pivkin, G. Karniadakis, Single-particle hydrodynamics in DPD: A new formulation, EPL 84 (2008) 10012.

[18] W. Pan, B. Caswell, G. Karniadakis, Rheology, microstructure and migration in Brownian colloidal suspensions, Langmuir 26 (2010) 133-142.

[19] W. Pan, B. Caswell, G. Karniadakis, A low-dimensional model for the red blood cell, Soft Matter 6 (2010) 4366-4376.

[20] S. K. Ranjith, B. S. V. Patnaik, S. Vedantam, Transport of DNA in hydrophobic microchannels: A dissipative particle dynamics simulation, Soft Matter 10 (2014) 4184-4191.

[21] V. Pryamitsyn, V. Ganesan, A coarse-grained explicit solvent simulation of rheology of colloidal suspensions, J. Chem. Phys. 122 (2005) 104906.

[22] B. Berne, P. Pechukas, Gaussian model potentials for molecular interactions, J. Chem. Phys. 56 (1972) 4213-4216.

[23] D. Liu, E. Keaveny, M. Maxey, G. Karniadakis, Force-coupling method for flows with ellipsoidal particles, J. Comput. Phys. 228 (2009) 3559-3581.

[24] M. Allen, G. Germano, Expressions for forces and torques in molecular simulations using rigid bodies, Molecular Physics 104 (2006) 3225-3235.

[25] J. Gay, B. Berne, Modification of the overlap potential to mimic a linear site-site potential, J. Chem. Phys. 74 (1981) 3316-3319.

[26] M. Babadi, M. Ejtehadi, R. Everaers, Analytical first derivatives of the REsquared interaction potential, J. Comput. Phys. 219 (2006) 770-779.

[27] R. Berardi, C. Fava, C. Zannoni, A generalized Gay-Berne intermolecular potential for biaxial particles, Chem. Phys. Lett. 236 (1995) 462-468.

[28] R. Berardi, C. Fava, C. Zannoni, A Gay-Berne potential for dissimilar biaxial particles, Chem. Phys. Lett. 297 (1998) 8-14. 
[29] R. Everaers, M. Ejtehadi, Interaction potentials for soft and hard ellipsoids, Phys. Rev. E 67 (2003) 041710.

[30] A. P. Cohen, E. Janai, E. Mogilko, A. B. Schofield, E. Sloutskin, Fluid suspensions of colloidal ellipsoids: Direct structural measurements, Phys. Rev. Lett. 107 (2011) 238301.

[31] M. Letz, A. Latz, Fluids of hard ellipsoids: Phase diagram including a nematic instability from Percus-Yevick theory, Phy. Rev. E 60 (1999) 5865-5871.

[32] E. Miguel, C. Vega, The global phase diagram of the Gay-Berne model, J. Chem. Phys. 117 (2002) 6313-6322.

[33] M. Wilson, Molecular dynamics simulations of flexible liquid crystal molecules using a Gay-Berne/Lennard-Jones model, J. Chem. Phys. 107 (1997) 8654-8663.

[34] R. Clift, J. Grace, M. Weber, Bubbles, drops, and particles, Dover Publications, 2005.

[35] Y. Han, A. Alsayed, M. Nobili, J. Zhang, T. Lubensky, A. Yodh, Brownian motion of an ellipsoid, Science 314 (2006) 626-630.

[36] Y. Han, A. Alsayed, M. Nobili, A. G. Yodh, Quasi-two-dimensional diffusion of single ellipsoids: Aspect ratio and confinement effects, Phys. Rev. E 80 (2009) 011403 .

[37] J. Happel, H. Brenner, Low Reynolds number hydrodynamics, Kluwer, Dordrecht, 1991.

[38] F. Perrin, Mouvement Brownien d'un ellipsoide - I. Dispersion diélectrique pour des molécules ellipsoidales, J. Phys. Radium 5 (1934) 497-511.

[39] F. Perrin, Mouvement Brownien d'un ellipsoide - II. Rotation libre et dépolarisation des fluorescences. Translation et diffusion de molécules ellipsoidales, J. Phys. Radium 7 (1936) 1-11.

[40] M. Rechtsman, F. Stillinger, S. Torquato, Designed interaction potentials via inverse methods for self-assembly, Phys. Rev. E 73 (2006) 011406.

[41] A. Chakrabarty, A. Konya, F. Wang, J. V. Selinger, K. Sun, Q. Wei, Brownian motion of boomerang colloidal particles, Phys. Rev. Lett. 111 (2013) 160603.

[42] H. Shen, Y. Li, P. Ren, D. Zhang, G. Li, Anisotropic coarse-grained model for proteins based on Gay-Berne and electric multipole potentials, J. Chem. Theory Comput. 10 (2014) 731-750. 